\title{
Impact of Chronic Inflammation, Assessed by hs-CRP, on the Association between Red Cell Distribution Width and Arterial Cardiovascular Disease: The Tromsø Study
}

Jostein Lappegård ${ }^{1}$ Trygve S. Ellingsen ${ }^{1} \quad$ Kristian Hindberg ${ }^{1} \quad$ Ellisiv B. Mathiesen ${ }^{1,2,3}$ Inger Njølstad ${ }^{1,4}$ Tom Wilsgaard ${ }^{4}$ Maja-Lisa Løchen ${ }^{4}$ Sigrid K. Brækkan ${ }^{1,5}$ John-Bjarne Hansen ${ }^{1,5}$

${ }^{1}$ K.G. Jebsen Thrombosis Research and Expertise Center (TREC), Department of Clinical Medicine, UiT The Arctic University of Norway, Tromsø, Norway

2 Brain and Circulation Research Group, Department of Clinical Medicine, UiT The Arctic University of Norway, Tromsø, Norway

${ }^{3}$ Department of Neurology and Neurophysiology, University Hospital of North Norway, Tromsø, Norway

${ }^{4}$ Department of Community Medicine, UiT The Arctic University of Norway, Tromsø, Norway

${ }^{5}$ Division of Internal Medicine, University Hospital of North Norway, Tromsø, Norway

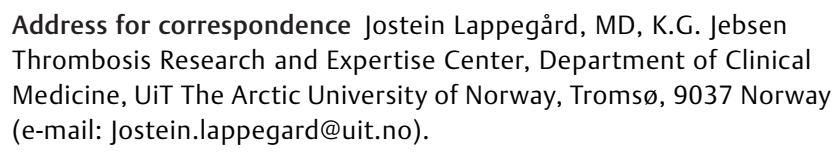
Thrombosis Research and Expertise Center, Department of Clinical Medicine, UiT The Arctic University of Norway, Tromsø, 9037 Norway (e-mail: Jostein.lappegard@uit.no).

TH Open 2018;2:e182-e189.

\begin{abstract}
Keywords

- epidemiology

- myocardial infarction

- stroke

- blood cells

- risk factors

Red cell distribution width (RDW), a measure of variability in size of circulating erythrocytes, is associated with arterial cardiovascular disease (CVD), but the underlying mechanism remains unclear. We aimed to investigate the impact of chronic inflammation as measured by high-sensitivity C-reactive protein (hs-CRP) on this relationship, and explore whether RDW could be a mediator in the causal pathway between inflammation and arterial CVD. Baseline characteristics, including RDW and hs-CRP, were obtained from 5,765 individuals attending a population-based cohort study. We followed up participants from inclusion in the fourth survey of the Troms $\varnothing$ Study (1994/1995) until December 31, 2012. Multivariable Cox-regression models were used to calculate hazard ratios (HR) with $95 \%$ confidence intervals (Cl) for incident myocardial infarction (MI) and ischemic stroke across quintiles of hs-CRP and RDW. Subjects with hs-CRP in the highest quintile had $44 \%$ higher risk of MI (HR: $1.44,95 \% \mathrm{Cl}$ : 1.14-1.80), and $64 \%$ higher risk of ischemic stroke (HR: $1.64,95 \% \mathrm{Cl}: 1.20-2.24)$ compared with subjects in the lowest quintile. RDW mediated 7.2\% (95\% Cl: 4.0-30.8\%) of the association between hs-CRP and ischemic stroke. Subjects with RDW in the highest quintile had $22 \%$ higher risk of MI (HR: $1.22,95 \% \mathrm{Cl}: 0.98-1.54)$ and $44 \%$ higher risk of ischemic stroke (HR: $1.44,95 \% \mathrm{Cl}: 1.06-1.97)$ compared with subjects in the lowest quintile. These risk estimates were slightly attenuated after adjustments for hsCRP. Our findings suggest that chronic inflammation is not a primary mechanism underlying the relationship between RDW and arterial CVD.
\end{abstract}

received

January 31, 2018 accepted after revision April 10, 2018
DOI https://doi.org/

10.1055/s-0038-1651523. ISSN 2512-9465. (c) 2018 Georg Thieme Verlag KG Stuttgart · New York
License terms

(c) (i) 


\section{Introduction}

Red blood cell distribution width (RDW) is a measure of the variability in size of circulating erythrocytes, and is reported by most automated blood cell counters. ${ }^{1}$ In combination with mean corpuscular volume (MCV), RDW has traditionally been used in the differential diagnosis of anemia. ${ }^{2}$ Various causes of premature release of red blood cells may lead to alterations in the RDW. ${ }^{3-5}$

Growing evidence supports a relationship between RDW and arterial cardiovascular disease (CVD). In two large population-based cohorts, RDW was associated with future risk of incident $\mathrm{MI}^{6,7}$ and ischemic stroke. ${ }^{8,9}$ However, the mechanisms behind these relationships are not clear.

Atherosclerosis, the principle cause of myocardial infarction (MI) and an important cause of ischemic stroke, ${ }^{10}$ is widely recognized as an inflammatory disease. ${ }^{11,12}$ C-reactive protein (CRP), an acute phase reactant synthesized in response to signals from macrophages and $T$ cells during inflammation (e.g., interleukin-6), ${ }^{13}$ is a predictor of MI and stroke. ${ }^{14-17}$ Inflammation affects red blood cell life span and modulates the effect of erythropoietin on the erythropoiesis, and can thereby affect RDW. ${ }^{18}$ Studies have reported an association between RDW and CRP, erythrocyte sedimentation rate (ESR), and interleukin-6. ${ }^{19-22}$

Since chronic inflammation is associated with increased risk of arterial CVD, and RDW is associated with several markers of chronic inflammation, it has been hypothesized that the apparent association between RDW and risk of arterial CVD could be explained by chronic inflammation. On one hand, chronic inflammation could merely serve as a confounder for the relationship between RDW and arterial CVD (-Fig. 1A). Alternatively, if RDW is causally associated with arterial CVD (e.g., through mechanisms of augmented erythrocyte aggregability or altered blood viscosity), it could act as mediator in the pathway between chronic inflammation and CVD (- Fig. 1B).

Despite the indications that chronic inflammation may influence the association between RDW and arterial CVD, this hypothesis has not been thoroughly investigated. Therefore, we set out to study whether the association between RDW and arterial CVD was confounded by chronic inflammation (assessed by hs-CRP), or whether chronic inflammation was associated with changes in RDW (mediator) that directly could lead to increased risk of arterial CVD.

\section{Materials and Methods}

\section{Study Population}

The fourth survey of the Tromsø study (Tromsø 4) was conducted in 1994-1995, and all inhabitants in the municipality of Tromsø, Norway, aged 25 or older, were invited to participate. A total of 27,158 individuals attended the first visit, yielding a participation rate of $77 \%$. Following this, all participants aged 55 to 74 years, as well as 5 to $8 \%$ randomly selected samples from each of the other 5-year age intervals (between 25-54 years and 75-85 years), were offered a follow-up visit (1994-1995). At this second visit, the parti-

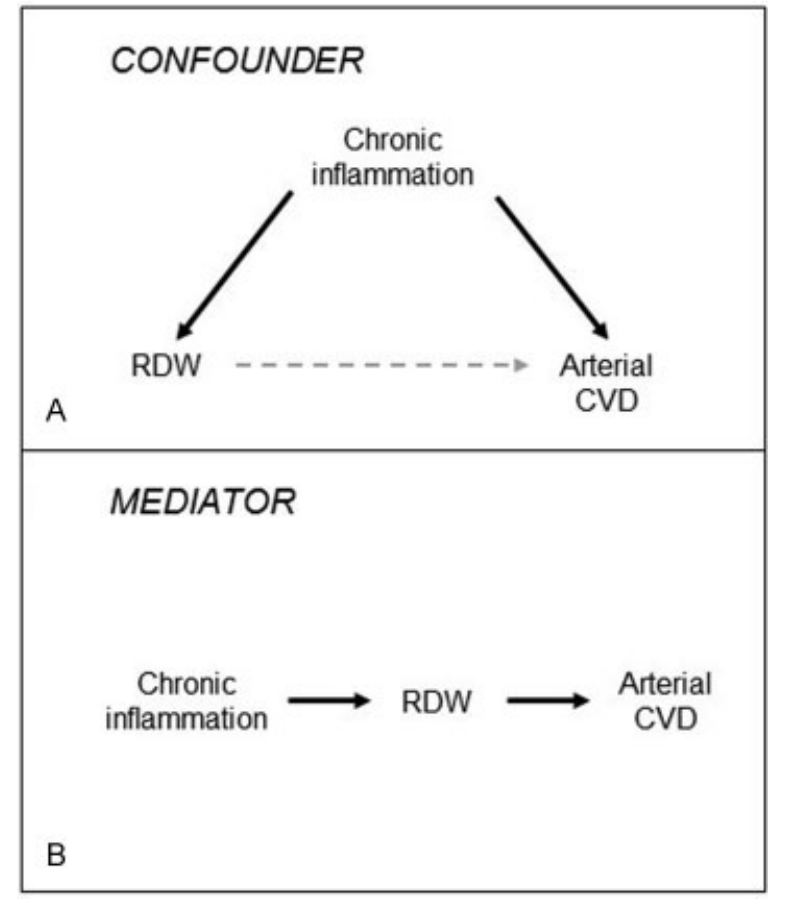

Fig. 1 Potential associations between chronic inflammation, red cell distribution width (RDW), and risk of cardiovascular disease (CVD). (A) Illustration of how chronic inflammation could act as a confounder of the association, affecting both RDW and risk of CVD. (B) Illustration of how RDW could act as a mediator in the causal pathway between chronic inflammation and arterial CVD.

cipants underwent a more thorough examination, and additional measurements including high-sensitivity CRP (hsCRP) were performed. All subjects attending the second visit of Tromsø 4 were enrolled in the present study ( $n=6,834$; $76 \%$ of those eligible). The study population has been described in detail elsewhere. ${ }^{23}$ We excluded subjects lacking measurements of RDW $(n=169)$ and hs-CRP $(n=118)$, subjects with a diagnosis of MI $(n=354)$ or stroke $(n=166)$ prior to enrolment, as well as subjects migrating from Tromsø before the second visit $(n=7)$. In addition, we excluded participants with hs-CRP levels $>10 \mathrm{mg} / \mathrm{L}$ ( $n=255)$ at baseline to avoid influence of possible acute phase reactions. This left us with 5,765 participants in the present study. The Regional Committee for Medical and Health Research Ethics approved the study, and informed written consent was acquired from all participants.

\section{Baseline Measurements}

Baseline information on each participant was obtained through self-administered questionnaires, blood samples, and physical examinations. Nonfasting blood samples were drawn from an antecubital vein into vacutainer tubes containing EDTA for anticoagulation (K3-EDTA $40 \mu \mathrm{L}, 0.37 \mathrm{~mol} / \mathrm{L}$ per tube). For the blood cell counts, including RDW, $5 \mathrm{~mL}$ of blood was analyzed within 12 hours in an automated blood cell counter (Coulter Counter; Coulter Electronics, Luton, UK). RDW was calculated by dividing the standard deviation (SD) of MCV with the MCV, then multiplying it by $100 .{ }^{24}$ For preparation of serum, the blood sample was given a 1-hour 
respite at room temperature before centrifugation. Hs-CRP was measured by a particle-enhanced immunoturbidimetric assay on a Modular P auto-analyzer (Roche/Hitachi) using reagents from Roche Diagnostics $\mathrm{GmbH}$, Mannheim, Germany. The lower detection limit for the assay was $0.03 \mathrm{mg} / \mathrm{L}$, and all measurements below this threshold were set to 0.03 $\mathrm{mg} / \mathrm{L}$. Daily changes in the assay precision for values between 0.1 and $20 \mathrm{mg} / \mathrm{L}$ were less than $4 \%$.

Trained personnel recorded blood pressure on the right upper arm with an automatic device (Dinamap Vital Signs Monitor; 1846, Critikon Inc., Tampa, Florida, United States). Starting with 2 minutes of rest in a seated position, three recordings with 2-minute intervals were completed. The mean value of the last two recordings was used in this article. Hypertension was defined as a systolic blood pressure $\geq 140 \mathrm{~mm} \mathrm{Hg}$, diastolic blood pressure $\geq 90 \mathrm{~mm} \mathrm{Hg}$, or current use of antihypertensive medication. Measurements of height and weight were performed using electronic scales, with participants wearing no shoes and light clothing. The body mass index (BMI) was calculated by dividing the weight in kilograms by the square of height in meters.

Information on diabetes and smoking was obtained from the questionnaires. The question on diabetes was stated as follows: "do you have or have you had diabetes?" (yes/no). Subjects were defined as non-smokers only if answering "no" to all three of the following questions: "do you smoke cigarettes daily?"; "do you smoke cigars/cigarillos daily?"; or "do you smoke pipe daily?" Study participants answering "yes" to any of the three were defined as daily smokers.

\section{Outcome Assessment}

All first-time events of MI and ischemic stroke were identified by searching a wide range of International Classification of Disease (ICD) codes at the University Hospital of North Norway and the National Causes of Death Registry at Statistics Norway. The Norwegian national 11-digit identification number allowed linkage to both diagnosis and death registries. The University Hospital of North Norway is the only hospital serving the municipality of Tromsø, and the next nearest hospital is around $250 \mathrm{~km}$ away. This ensured that very few cases were lost during follow-up. An independent endpoint committee validated all stroke and MI events by retrieval of hospital medical records, death certificates, autopsy reports, and records from nursing homes, ambulance services, and general practitioners. The thorough process of identification and validation of outcomes has been previously described in detail. ${ }^{6,9}$

\section{Statistical Analyses}

Statistical analyses were performed with STATA version 14.0 (StataCorp LLC, College Station, Texas, United States). Followup time was calculated from the date of enrolment in Troms $\emptyset$ 4 (1994-1995) to the date of a stroke or MI event, date of death, date of migration from Tromsø, or until the end of follow-up (December 31, 2012), whichever came first. A total of 115 subjects experienced both outcomes during followup, and were censored from the first occurring event.

The study population was categorized into quintiles according to their baseline RDW values (Quintile 1: $\leq 12.4 \%$, Quintile 2:
12.5-12.8\%, Quintile 3: 12.9-13.1\%, Quintile 4: 13.2-13.5\%, Quintile 5: $\geq 13.6 \%$ ) and their baseline hs-CRP values (Quintile 1: 0.01-0.51 mg/L, Quintile 2: 0.52-0.88 mg/L, Quintile 3: 0.89$1.47 \mathrm{mg} / \mathrm{L}$, Quintile 4: 1.48-2.68 mg/L, Quintile 5: 2.69-9.97 $\mathrm{mg} / \mathrm{L})$. Age-adjusted baseline characteristics across quintiles of RDW and hs-CRP were estimated using analysis of covariance (ANCOVA). The correlation between RDW and hs-CRP was evaluated by calculating crude Pearson's correlation coefficients.

Crude incidence rates (IRs) were calculated as the total number of events divided by the total person time, and expressed as number of events per 1,000 person-years at risk. Cox proportional hazard regression models were used to estimate hazard ratios (HRs) with $95 \%$ confidence intervals (CI) for MI and ischemic stroke across quintiles of RDW and hs-CRP, and per $1 \mathrm{SD}$ increment in $\mathrm{RDW}(0.8 \%)$ and hs-CRP $(1.69 \mathrm{mg} / \mathrm{L})$. Subjects in Quintile 1 of each exposure group were used as reference in the categorical analyses. Age was used as time scale in the regression analyses. The multivariable analyses were divided into three different models. Model 1 included adjustments for age (through the time scale), sex, and BMI. Model 2 included the covariates in model 1 , in addition to smoking status, platelet count, hypertension, total cholesterol, triglycerides, and self-reported diabetes. In the analyses across quintiles of RDW, hs-CRP was added as a covariate to adjustment model 2 , to evaluate whether chronic inflammation confounded the association between RDW and the CVD outcomes. In the analyses across quintiles of hs-CRP, baseline RDW values were added to adjustment model 2 , to evaluate the potential mediating role of RDW. Furthermore, mediation analyses were conducted to calculate the proportion of the total exposureoutcome effect that could be due to the mediator. ${ }^{25}$

\section{Results}

There were 913 incident MIs (IR: 11.5 per 1,000 personyears, 95\% CI: 10.7-12.2) and 461 incident ischemic stroke events (IR: 5.8 per 1,000 person-years, 95\% CI: 5.3-6.3) during a median follow-up of 17.6 years. - Table 1 shows crude baseline characteristics according to subjects who experienced MI, subjects who experienced ischemic stroke, and those who remained free of arterial CVD throughout the study period. The proportion of men, subjects with diabetes and hypertension, as well as age, levels of triglycerides, RDW, hs-CRP, hemoglobin, and BMI were all higher in those with than those without arterial CVD. The proportion of smokers was higher among those suffering an MI, but not among those with ischemic stroke. The crude correlation analysis revealed a low, but statistically significant correlation coefficient between hs-CRP and RDW $(r=0.13, p<0.0001)$. Ageadjusted baseline characteristics across quintiles of RDW and hs-CRP are shown in - Supplementary Table S1.

- Table 2 displays IRs and HRs for MI and ischemic stroke across quintiles of hs-CRP. The crude IRs of both MI and ischemic stroke increased with increasing serum concentrations of hs-CRP. In multivariable-adjusted analyses, subjects with hs-CRP in the upper quintile had a $44 \%$ higher risk of MI (model 2, HR: 1.44, 95\% CI: 1.14-1.80) and 64\% higher risk of 
Table 1 Baseline characteristics stratified according to future development of myocardial infarction or ischemic stroke (The Tromsø Study)

\begin{tabular}{|l|l|l|l|}
\hline & No event & $\begin{array}{l}\text { Myocardial } \\
\text { infarction }\end{array}$ & $\begin{array}{l}\text { Ischemic } \\
\text { stroke }\end{array}$ \\
\hline$N$ & 4,506 & 913 & 461 \\
\hline RDW, \% & $13.0 \pm 0.8$ & $13.1 \pm 0.8$ & $13.2 \pm 1.0$ \\
\hline hs-CRP, mg/L & $1.67 \pm 1.69$ & $2.11 \pm 1.90$ & $2.17 \pm 1.86$ \\
\hline Age, y & $58.4 \pm 10.5$ & $63.8 \pm 7.5$ & $65.3 \pm 6.8$ \\
\hline Sex, \% males & $44.4(2001)$ & $59.5(543)$ & $53.8(248)$ \\
\hline $\begin{array}{l}\text { Body mass } \\
\text { index kg/m }\end{array}$ & $25.7 \pm 3.9$ & $26.6 \pm 4.1$ & $26.4 \pm 3.9$ \\
\hline Daily smoking, \% & $32.0(1,440)$ & $38.2(349)$ & $31.0(143)$ \\
\hline $\begin{array}{l}\text { Hemoglobin } \\
\text { total), g/dL }\end{array}$ & $14.1 \pm 1.1$ & $14.4 \pm 1.0$ & $14.3 \pm 1.1$ \\
\hline $\begin{array}{l}\text { Thrombocytes, } \\
\times 10^{9} / \mathrm{L}\end{array}$ & $248 \pm 56$ & $246 \pm 56$ & $247 \pm 55$ \\
\hline Hypertension, \% & $50.8(2,288)$ & $72.8(665)$ & $77.2(356)$ \\
\hline $\begin{array}{l}\text { Total cholesterol, } \\
\text { mmol/L }\end{array}$ & $6.7 \pm 1.3$ & $7.0 \pm 1.2$ & $6.8 \pm 1.3$ \\
\hline $\begin{array}{l}\text { Triglycerides, } \\
\text { mmol/L }\end{array}$ & $1.6 \pm 1.0$ & $1.9 \pm 1.1$ & $1.8 \pm 1.0$ \\
\hline $\begin{array}{l}\text { Red blood cells, } \\
\times 10^{12} / \mathrm{L}\end{array}$ & $4.6 \pm 0.4$ & $4.7 \pm 0.4$ & $4.7 \pm 0.4$ \\
\hline $\begin{array}{l}\text { Self-reported } \\
\text { diabetes, \% }\end{array}$ & $1.9(85)$ & $5.6(51)$ & $6.5(30)$ \\
\hline
\end{tabular}

Abbreviations: hs-CRP, high-sensitivity C-reactive protein; RDW, red cell distribution width.

Note: Baseline characteristics of study participants stratified according to future development of myocardial infarction or ischemic stroke. The values are reported as means \pm standard deviation or as percentages with number in brackets.

ischemic stroke (model 2, HR: 1.64, 95\% CI: 1.20-2.24) compared with subjects with hs-CRP in the lowest quintile. The risk estimate for MI was essentially unchanged after including RDW in the adjustment model (model 3, HR: 1.42, 95\% CI: 1.13-1.78), whereas the risk estimate for ischemic stroke was modestly attenuated (model 3, HR: $1.59,95 \% \mathrm{CI}$ : 1.16-2.17). In mediation analyses, RDW was estimated to mediate $7.2 \%$ (95\% CI: $4.0-30.8 \%$ ) of the total association between hs-CRP and ischemic stroke. The association between hs-CRP and MI was not mediated by RDW $(0.6 \%$, 95\% CI: -1.4 to $4.3 \%$ ). In the analysis of CRP as a continuous variable, $1 \mathrm{SD}(1.69 \mathrm{mg} / \mathrm{L})$ increase in hs-CRP was associated with an $8 \%$ higher risk of MI (model 2, HR: $1.08,95 \%$ CI: $1.04-$ 1.12 ) and an $11 \%$ higher risk of ischemic stroke (model 2 , HR: 1.11, 95\% CI: 1.06-1.16).

HRs and IRs for MI and ischemic stroke across quintiles of RDW are shown in - Table 3. In the multivariable model, subjects with RDW values in the highest quintile had a $22 \%$ higher risk of MI (HR: $1.22,95 \% \mathrm{CI}: 0.98-1.54$ ) and a $44 \%$ higher risk of ischemic stroke (HR: $1.44,95 \%$ CI: 1.06-1.97) compared with the reference group. After addition of hs-CRP to the multivariable analyses, the risk estimates were slightly attenuated for both outcomes, with a HR for MI of 1.17 (model 3, 95\% CI: 0.93-1.47), and a HR for ischemic stroke of 1.37 (model 3, 95\% CI: 1.00-1.87). When modeled as a continuous variable, a $1 \mathrm{SD}$ increase $(0.8 \%)$ in $\mathrm{RDW}$ was associated with a $6 \%$ higher risk of MI (model 2, HR: 1.06, 95\% CI: $0.99-1.14)$ and a $14 \%$ higher risk of ischemic stroke (model 2, HR: 1.14, 95\% CI: 1.05-1.24). These risk estimates were also slightly attenuated by adding hs-CRP to the multivariable analysis (model 3).

\section{Discussion}

In the present study, we explored the impact of chronic inflammation on the association between RDW and arterial CVD in a prospective, population-based cohort. RDW was associated with higher risks of MI and ischemic stroke, and these risk estimates were only modestly affected by adjustment for hs-CRP. Hs-CRP was associated with MI and ischemic stroke independent of other traditional atherosclerotic risk factors. Addition of RDW to the multivariable models slightly attenuated the association between hs-CRP and ischemic stroke, and specific mediation analyses indicated a modest role of RDW in mediating this relationship. Our findings suggest that chronic inflammation had only a modest impact on the relationship between RDW and arterial CVD.

CRP is a well-documented risk factor for arterial CVD. In a meta-analysis of 54 long-term prospective studies including 160,309 subjects without previous vascular disease, the risk ratio per $1 \mathrm{SD}$ increase in CRP was 1.37 for coronary heart disease, and 1.27 for ischemic stroke. ${ }^{17}$ Accordingly, we found an association between hs-CRP and both MI and ischemic stroke in our study. Furthermore, our results also concurred with most previous studies on the relationship between RDW and arterial CVD. In the Malmö Diet and Cancer study, a cohort with RDW measurements of 26,879 participants, Söderholm et al found a 30\% higher risk of cerebral infarction for those in the highest versus the lowest RDW quartile. ${ }^{8}$ In the same cohort, subjects in the highest RDW quartile had an $82 \%$ higher risk of fatal coronary events, but not increased risk of nonfatal coronary events (HR: 0.96, 95\% CI: 0.82-1.12), compared with those in the lowest quartile. ${ }^{7}$ In previous studies using the phase 1 population from Tromsø $4(n>26,000)$, subjects with RDW in the highest quintile had $30 \%$ higher risk of ischemic stroke, ${ }^{9}$ and $34 \%$ higher risk of MI compared with subjects in the lowest quintile. ${ }^{6}$ Results from a prospective study on 3,226 previously healthy subjects from Taiwan, ${ }^{26}$ however, reported no relationship between RDW and incident MI.

Several studies have reported a relationship between various markers of inflammation and RDW. Chronic diseases and inflammation affect the erythropoiesis and modulate red cell life span, and can thereby cause changes in the RDW. ${ }^{18}$ In a cohort of 3,845 adult outpatients, Lippi et al found a threefold higher ESR and CRP in subjects with RDW in the highest compared with the lowest quartile. ${ }^{19}$ A correlation between serum levels of CRP and RDW has been described in patients with non-dipper hypertension $(r=0.403, p<0.001),{ }^{27}$ patients with coronary artery disease $(r=0.181, p<0.001),{ }^{22}$ and in overweight adolescents 
Table 2 Adjusted HRs for myocardial infarction and ischemic stroke according to categories of hs-CRP (The Tromsø Study)

\begin{tabular}{|c|c|c|c|c|c|c|}
\hline hs-CRP & Events & $\begin{array}{l}\text { Crude IR } \\
(95 \% \mathrm{CI})\end{array}$ & $\begin{array}{l}\text { Age/Sex-adjusted } \\
\text { HR (95\% CI) }\end{array}$ & $\begin{array}{l}\text { Model } 1 \\
\text { HR }(95 \% \mathrm{Cl})\end{array}$ & $\begin{array}{l}\text { Model } 2 \\
\text { HR (95\% Cl) }\end{array}$ & $\begin{array}{l}\text { Model } 3 \\
\text { HR (95\% CI) }\end{array}$ \\
\hline \multicolumn{7}{|c|}{ Myocardial infarction } \\
\hline Q1 & 126 & $7.2(6.0-8.6)$ & Ref. & Ref. & Ref. & Ref. \\
\hline Q2 & 153 & $9.3(8.0-10.9)$ & $1.08(0.85-1.36)$ & $1.05(0.83-1.33)$ & $0.97(0.77-1.24)$ & $0.97(0.76-1.23$ \\
\hline Q3 & 195 & $12.0(10.4-13.8)$ & $1.33(1.06-1.67)$ & $1.26(1.00-1.58)$ & $1.11(0.88-1.39)$ & $1.10(0.87-1.39$ \\
\hline Q4 & 196 & $12.6(10.9-14.5)$ & $1.39(1.11-1.74)$ & $1.30(1.04-1.63)$ & $1.10(0.87-1.39)$ & $1.09(0.86-1.38$ \\
\hline Q5 & 243 & $17.4(15.4-19.8)$ & $1.94(1.56-2.40)$ & $1.81(1.45-2.25)$ & $1.44(1.14-1.80)$ & $1.42(1.13-1.78$ \\
\hline $\begin{array}{l}\text { Per } 1 \text { SD } \\
\text { increase }\end{array}$ & 913 & $11.5(10.7-12.2)$ & $1.12(1.09-1.16)$ & $1.11(1.08-1.15)$ & $1.08(1.04-1.12)$ & $1.08(1.04-1.11$ \\
\hline \multicolumn{7}{|c|}{ Ischemic stroke } \\
\hline Q1 & 65 & $3.7(2.9-4.7)$ & Ref. & Ref. & Ref. & Ref. \\
\hline Q2 & 73 & $4.4(3.5-5.6)$ & $0.98(0.70-1.37)$ & $0.96(0.69-1.35)$ & $0.87(0.62-1.23)$ & $0.87(0.62-1.22$ \\
\hline Q3 & 90 & $5.5(4.5-6.8)$ & $1.15(0.84-1.59)$ & $1.12(0.81-1.55)$ & $1.00(0.72-1.38)$ & $0.99(0.71-1.37$ \\
\hline Q4 & 97 & $6.2(5.1-7.6)$ & $1.31(0.95-1.79)$ & $1.27(0.92-1.74)$ & $1.13(0.82-1.57)$ & $1.11(0.80-1.54$ \\
\hline Q5 & 136 & $9.8(8.2-11.5)$ & $2.06(1.53-2.77)$ & $2.00(1.48-2.70)$ & $1.64(1.20-2.24)$ & $1.59(1.16-2.17$ \\
\hline $\begin{array}{l}\text { Per } 1 \text { SD } \\
\text { increase }\end{array}$ & 461 & $5.8(5.3-6.3)$ & $1.14(1.09-1.19)$ & $1.14(1.09-1.19)$ & $1.11(1.06-1.16)$ & $1.10(1.05-1.16$ \\
\hline
\end{tabular}

Abbreviations: BMI, body mass index; $\mathrm{Cl}$, confidence interval; hs-CRP, high-sensitivity C-reactive protein; HR, hazard ratio; IR, incidence rate; RDW, red cell distribution width; SD, standard deviation.

Notes:

Model 1: Age, sex, BMI.

Model 2: Model $1+$ smoking, platelet count, hypertension, total cholesterol, triglycerides, self-reported diabetes.

Model 3: Model $2+$ RDW.

IRs per 1,000 person-years and adjusted HRs with $95 \% \mathrm{Cl}$ for myocardial infarction and ischemic stroke across quintiles of hs-CRP, and per 1 SD increase in hs-CRP.

Table 3 Adjusted HRs for myocardial infarction and ischemic stroke according to categories of RDW (The Tromsø Study)

\begin{tabular}{|c|c|c|c|c|c|c|}
\hline RDW & Events & $\begin{array}{l}\text { Crude IR } \\
(95 \% \mathrm{CI})\end{array}$ & $\begin{array}{l}\text { Age/Sex-adjusted } \\
\text { HR }(95 \% \mathrm{Cl})\end{array}$ & $\begin{array}{l}\text { Model } 1 \\
\text { HR }(95 \% \mathrm{Cl})\end{array}$ & $\begin{array}{l}\text { Model } 2 \\
\text { HR }(95 \% \mathrm{Cl})\end{array}$ & $\begin{array}{l}\text { Model } 3 \\
\text { HR }(95 \% \mathrm{Cl})\end{array}$ \\
\hline \multicolumn{7}{|c|}{ Myocardial infarction } \\
\hline Q1 & 138 & $8.0(6.8-9.4)$ & Ref. & Ref. & Ref. & Ref. \\
\hline Q2 & 198 & $9.5(8.2-10.9)$ & $1.01(0.81-1.25)$ & $1.00(0.80-1.24)$ & $0.99(0.79-1.24)$ & $0.98(0.79-1.23)$ \\
\hline Q3 & 184 & $12.6(10.9-14.6)$ & $1.23(0.99-1.54)$ & $1.22(0.98-1.53)$ & $1.19(0.95-1.49)$ & $1.18(0.94-1.47)$ \\
\hline Q4 & 190 & $13.8(12.0-15.9)$ & $1.30(1.04-1.62)$ & $1.29(1.04-1.61)$ & $1.22(0.97-1.53)$ & $1.20(0.95-1.50)$ \\
\hline Q5 & 203 & $15.4(13.4-17.6)$ & $1.41(1.13-1.75)$ & $1.40(1.13-1.74)$ & $1.22(0.98-1.54)$ & $1.17(0.93-1.47)$ \\
\hline $\begin{array}{l}\text { Per } 1 \text { SD } \\
\text { increase }\end{array}$ & 913 & $11.5(10.7-12.2)$ & $1.11(1.04-1.18)$ & $1.11(1.04-1.18)$ & $1.06(0.99-1.14)$ & $1.04(0.97-1.12)$ \\
\hline \multicolumn{7}{|c|}{ Ischemic stroke } \\
\hline Q1 & 70 & $4.0(3.2-5.1)$ & Ref. & Ref. & Ref & Ref. \\
\hline Q2 & 97 & $4.6(3.8-5.7)$ & $0.97(0.71-1.32)$ & $0.95(0.70-1.30)$ & $0.98(0.71-1.33)$ & $0.97(0.71-1.32)$ \\
\hline Q3 & 86 & $5.9(4.8-7.3)$ & $1.11(0.81-1.53)$ & $1.11(0.81-1.52)$ & $1.12(0.81-1.54)$ & $1.11(0.80-1.53)$ \\
\hline Q4 & 89 & $6.5(5.3-8.0)$ & $1.17(0.86-1.61)$ & $1.18(0.86-1.61)$ & $1.22(0.88-1.69)$ & $1.19(0.86-1.64)$ \\
\hline Q5 & 119 & $9.0(7.5-10.8)$ & $1.57(1.17-2.11)$ & $1.57(1.16-2.11)$ & $1.44(1.06-1.97)$ & $1.37(1.00-1.87)$ \\
\hline $\begin{array}{l}\text { Per } 1 \text { SD } \\
\text { increase }\end{array}$ & 461 & $5.8(5.3-6.3)$ & $1.17(1.09-1.26)$ & $1.18(1.09-1.27)$ & $1.14(1.05-1.24)$ & $1.11(1.03-1.21)$ \\
\hline
\end{tabular}

Abbreviations: BMI, body mass index; $\mathrm{Cl}$, confidence interval; hs-CRP, high-sensitivity C-reactive protein; HR, hazard ratio; IR, incidence rate; RDW, red cell distribution width; SD, standard deviation.

Notes:

Model 1: Age, sex, BMI.

Model 2: Model $1+$ smoking, platelet count, hypertension, total cholesterol, triglycerides, self-reported diabetes.

Model 3: Model $2+$ hs-CRP.

IRs per 1,000 person-years and adjusted $\mathrm{HRs}$ with $95 \% \mathrm{Cl}$ for myocardial infarction and ischemic stroke across quintiles of RDW, and per $1 \mathrm{SD}$ increase in RDW. 
$(r=0.241, p=0.034) .{ }^{28}$ In patients with Alzheimer's disease, both CRP $(r=0.350, p<0.001)$ and ESR $(r=0.457$, $p<0.001$ ) correlated with RDW. ${ }^{29}$

Previously, only a few studies have investigated the potential impact of chronic inflammation as a confounder of the relationship between RDW and adverse outcomes. In the Malmö Diet and Cancer study, the association between RDW and fatal coronary events remained unchanged after addition of leukocyte count to the multivariable analyses. ${ }^{7}$ Similarly, the association between RDW and heart failure did not change after adjustments for CRP in a large prospective study, ${ }^{30}$ and a $1 \%$ increment in RDW yielded equal risk of allcause mortality independent of hs-CRP levels. ${ }^{31}$

Our findings undermine the hypothesis that chronic inflammation is the main explanation for the observed relationship between RDW and risk of arterial CVD. First, RDW and hs-CRP levels were weakly correlated in our population. Second, the relationship between RDW and arterial CVD was only modestly affected by adding hs-CRP to the multivariable analyses. However, residual confounding by inflammation cannot be completely ruled out, as our measurements of hs-CRP might not sufficiently reflect a chronic inflammatory state. Nevertheless, if the effect of RDW on arterial CVD is indirect, residual confounding by a factor other than inflammation seems more plausible in the light of our findings. Oxidative stress, stimulated by common cardiovascular risk factors, plays a crucial role in the pathogenesis of atherosclerosis. ${ }^{32,33}$ Oxidative stress is also an important regulator of hematopoietic cell homeostasis, and could thereby influence RDW and confound the relationship between RDW and arterial CVD. ${ }^{34}$ Unfortunately, we do not have measurements of reactive oxygen species in our population, and we were unable to adjust for this potential confounder. Anemia, shown to be a risk factor for $\mathrm{CVD},{ }^{35}$ could also potentially confound the association, as it is clearly linked to RDW. However, several prospective studies, including previous analyses of the Tromsø study, have shown that the association between RDW and arterial CVD is independent of anemia. $6,8,9,36$

A direct relationship between erythrocyte size variability and arterial CVD may be biologically plausible. Erythrocytes play an important role in atherosclerotic plaque development and stability. Deposition of the free cholesterol contained in the erythrocyte membrane is considered an important contributor to atherosclerotic plaque growth, ${ }^{37,38}$ and atherosclerotic plaques susceptible to rupture tend to have a higher lipid content than stable plaques. ${ }^{39}$ RDW is associated with a higher cholesterol content in the erythrocyte membranes, ${ }^{40}$ and intraplaque hemorrhage could thereby have a greater impact on plaque growth and instability in subjects with high RDW. Furthermore, RDW is associated with reduced red cell deformability, ${ }^{41}$ which again is linked to increased aggregation of erythrocytes, altered blood viscosity, and impaired blood flow in the microcirculation. ${ }^{42-44}$ RDW is also associated with venous thromboembolism, a disease in which mechanism of hypercoagulability and stasis are central players. Our results point toward a modest and direct role of RDW in the development of ischemic stroke, but not in the development of MI. Growing evidence supports more pronounced roles for hypercoagulability and stasis in the pathogenesis of ischemic stroke than in the pathogenesis of MI. First, mechanisms related to hypercoagulability, such as prothrombotic genotypes, are reported to be more pronounced in ischemic stroke than in MI. ${ }^{45}$ Second, approximately $40 \%$ of the ischemic strokes are caused by cardioembolic events, in which stasis plays a pivotal role in the pathogenesis. ${ }^{46}$

A major strength of our study is the prospective design with a long follow-up time. Participants were recruited from the general population with a high attendance rate. Few cases were missed during follow-up due to the single hospital serving the Tromsø area, and the thorough process of case identification and validation. One limitation to the study is the skewed age distribution due to the selected population invited to the second visit of Tromsø 4, which may reduce the generalizability of our findings. There was only one measurement of hs-CRP and RDW throughout the study period, which could result in an underestimation of the true associations due to regression dilution bias. ${ }^{47}$ Another potential weakness with only one measurement is the uncertainty of whether increased hs-CRP levels could be due to a chronic or an acute inflammatory process. To avoid this, all subjects with hs-CRP $>10 \mathrm{mg} / \mathrm{L}$ were excluded from our analyses, as this indicates an ongoing acute-phase response. ${ }^{48}$

In conclusion, we found that the association between RDW and arterial CVD was not confounded by hs-CRP, and that RDW had a modest direct effect on the risk of ischemic stroke, but not MI. Our study calls for a reassessment of chronic inflammation as the main explanation for the association between RDW and arterial CVD. Future studies are warranted to further explore other potentially underlying mechanisms.

\section{Funding}

K.G. Jebsen Thrombosis Research and Expertise Centre (TREC) is supported by an independent grant from Stiftelsen Kristian Gerhard Jebsen. S.K.B. and J-B.H. have received research grants from the Northern Norway Regional Health Authority (URL: http://www.helsenord.no/). The funders had no role in study design, data collection and analysis, decision to publish, or preparation of the manuscript.

\section{Authors' Contributions}

J.L.-analyzed the data and drafted the manuscript.

T.S.E.-interpreted the results and revised the manuscript. K.H.-provided statistical support, interpreted the results, and revised the manuscript.

E.B.M.-collected data, interpreted the results, and revised the manuscript.

I.N.-collected data, interpreted the results, and revised the manuscript.

T.W.-collected data and revised the manuscript.

M-L.L.-collected data, interpreted the results, and revised the manuscript.

S.K.B.-designed the study, interpreted the results, and revised the manuscript. 
J-B.H.-designed the study, interpreted the results, and revised the manuscript.

\section{Conflict of Interest \\ None declared.}

\section{Acknowledgments}

The authors thank participants of The Tromsø Study for their important contributions.

\section{References}

1 Simel DL, DeLong ER, Feussner JR, Weinberg JB, Crawford J. Erythrocyte anisocytosis. Visual inspection of blood films vs automated analysis of red blood cell distribution width. Arch Intern Med 1988; 148(04):822-824

2 Bessman JD, Gilmer PR Jr, Gardner FH. Improved classification of anemias by MCV and RDW. Am J Clin Pathol 1983;80(03):322-326

3 Rodak BF, Fritsma GA, Doig K. Hematology: Clinical Principles and Applications. Philadelphia, PA: Saunders Elsevier; 2007

4 Monzon CM, Beaver BD, Dillon TD. Evaluation of erythrocyte disorders with mean corpuscular volume $(\mathrm{MCV})$ and red cell distribution width (RDW). Clin Pediatr (Phila) 1987;26(12): 632-638

5 Nagajothi N, Braverman A. Elevated red cell distribution width in the diagnosis of thrombotic thrombocytopenic purpura in patients presenting with anemia and thrombocytopenia. South Med J 2007;100(03):257-259

6 Skjelbakken T, Lappegård J, Ellingsen TS, et al. Red cell distribution width is associated with incident myocardial infarction in a general population: the Tromsø Study. J Am Heart Assoc 2014;3 (04):e001109

7 Borné Y, Smith JG, Melander O, Engström G. Red cell distribution width in relation to incidence of coronary events and case fatality rates: a population-based cohort study. Heart 2014;100(14): 1119-1124

8 Söderholm M, Borné Y, Hedblad B, Persson M, Engström G. Red cell distribution width in relation to incidence of stroke and carotid atherosclerosis: a population-based cohort study. PLoS One 2015;10(05):e0124957

9 Lappegård J, Ellingsen TS, Skjelbakken T, et al. Red cell distribution width is associated with future risk of incident stroke. The Tromsø Study. Thromb Haemost 2016;115(01):126-134

10 Libby P. Current concepts of the pathogenesis of the acute coronary syndromes. Circulation 2001;104(03):365-372

11 Libby P. Inflammation in atherosclerosis. Nature 2002;420 (6917):868-874

12 Ross R. Atherosclerosis-an inflammatory disease. N Engl J Med 1999;340(02):115-126

13 Gabay C, Kushner I. Acute-phase proteins and other systemic responses to inflammation. N Engl J Med 1999;340(06):448-454

14 Ridker PM, Cushman M, Stampfer MJ, Tracy RP, Hennekens CH. Inflammation, aspirin, and the risk of cardiovascular disease in apparently healthy men. N Engl J Med 1997;336(14):973-979

15 Ridker PM, Glynn RJ, Hennekens CH. C-reactive protein adds to the predictive value of total and HDL cholesterol in determining risk of first myocardial infarction. Circulation 1998;97(20):2007-2011

16 Danesh J, Collins R, Appleby P, Peto R. Association of fibrinogen, Creactive protein, albumin, or leukocyte count with coronary heart disease: meta-analyses of prospective studies. JAMA 1998;279 (18):1477-1482

17 Kaptoge S, Di Angelantonio E, Lowe G, et al; Emerging Risk Factors Collaboration. C-reactive protein concentration and risk of coronary heart disease, stroke, and mortality: an individual participant meta-analysis. Lancet 2010;375(9709):132-140
18 Weiss G, Goodnough LT. Anemia of chronic disease. N Engl J Med 2005;352(10):1011-1023

19 Lippi G, Targher G, Montagnana M, Salvagno GL, Zoppini G, Guidi GC. Relation between red blood cell distribution width and inflammatory biomarkers in a large cohort of unselected outpatients. Arch Pathol Lab Med 2009;133(04):628-632

20 Förhécz Z, Gombos T, Borgulya G, Pozsonyi Z, Prohászka Z, Jánoskuti L. Red cell distribution width in heart failure: prediction of clinical events and relationship with markers of ineffective erythropoiesis, inflammation, renal function, and nutritional state. Am Heart J 2009;158(04):659-666

21 Miyamoto K, Inai K, Takeuchi D, Shinohara T, Nakanishi T. Relationships among red cell distribution width, anemia, and interleukin-6 in adult congenital heart disease. Circ J 2015;79 (05):1100-1106

22 Lappé JM, Horne BD, Shah SH, et al. Red cell distribution width, Creactive protein, the complete blood count, and mortality in patients with coronary disease and a normal comparison population. Clin Chim Acta 2011;412(23-24):2094-2099

23 Jacobsen BK, Eggen AE, Mathiesen EB, Wilsgaard T, Njølstad I. Cohort profile: the Tromso Study. Int J Epidemiol 2012;41(04): 961-967

24 Caporal FA, Comar SR. Evaluation of RDW-CV, RDW-SD, and MATH-1SD for the detection of erythrocyte anisocytosis observed by optical microscopy. J Bras Patol Med Lab 2013;49:324-331

25 Hicks R, Tingley D. Causal mediation analysis. Stata J 2011; 11:605-619

26 Chen P-C, Sung F-C, Chien K-L, Hsu H-C, Su T-C, Lee Y-T. Red blood cell distribution width and risk of cardiovascular events and mortality in a community cohort in Taiwan. Am J Epidemiol 2010;171(02):214-220

27 Özcan F, Turak O, Durak A, et al. Red cell distribution width and inflammation in patients with non-dipper hypertension. Blood Press 2013;22(02):80-85

28 Fujita B, Strodthoff D, Fritzenwanger M, et al. Altered red blood cell distribution width in overweight adolescents and its association with markers of inflammation. Pediatr Obes 2013;8(05): 385-391

29 Öztürk ZA, Ünal A, Yiğiter R, et al. Is increased red cell distribution width (RDW) indicating the inflammation in Alzheimer's disease (AD)? Arch Gerontol Geriatr 2013;56(01):50-54

30 Emans ME, Gaillard CA, Pfister R, et al. Red cell distribution width is associated with physical inactivity and heart failure, independent of established risk factors, inflammation or iron metabolism; the EPIC-Norfolk study. Int J Cardiol 2013;168(04):3550-3555

31 Horne BD, Muhlestein JB, Bennett ST, Anderson JL. The red cell distribution width predicts mortality among patients free from systemic inflammation. Circulation 2014;130:A14819

32 Vogiatzi G, Tousoulis D, Stefanadis C. The role of oxidative stress in atherosclerosis. Hellenic J Cardiol 2009;50(05):402-409

33 Deanfield JE, Halcox JP, Rabelink TJ. Endothelial function and dysfunction: testing and clinical relevance. Circulation 2007; 115(10):1285-1295

34 Ghaffari S. Oxidative stress in the regulation of normal and neoplastic hematopoiesis. Antioxid Redox Signal 2008;10(11): 1923-1940

35 Sarnak MJ, Tighiouart H, Manjunath G, et al. Anemia as a risk factor for cardiovascular disease in The Atherosclerosis Risk in Communities (ARIC) study. J Am Coll Cardiol 2002;40(01):27-33

36 Lappegård J, Ellingsen TS, Vik A, et al. Red cell distribution width and carotid atherosclerosis progression. The Tromsø Study. Thromb Haemost 2015;113(03):649-654

37 Kolodgie FD, Gold HK, Burke AP, et al. Intraplaque hemorrhage and progression of coronary atheroma. N Engl J Med 2003;349(24): 2316-2325

38 Kolodgie FD, Burke AP, Nakazawa G, Cheng Q, Xu X, Virmani R. Free cholesterol in atherosclerotic plaques: where does it come from? Curr Opin Lipidol 2007;18(05):500-507 
39 Felton CV, Crook D, Davies MJ, Oliver MF. Relation of plaque lipid composition and morphology to the stability of human aortic plaques. Arterioscler Thromb Vasc Biol 1997;17(07): 1337-1345

40 Tziakas D, Chalikias G, Grapsa A, Gioka T, Tentes I, Konstantinides $S$. Red blood cell distribution width: a strong prognostic marker in cardiovascular disease: is associated with cholesterol content of erythrocyte membrane. Clin Hemorheol Microcirc 2012;51(04): 243-254

41 Patel K, Mohanty J, Kanapuru B, Hesdorffer C, Ershler W, Rifkind J. Association of the red cell distribution width with red blood cell deformability. In: Welch WJ, Palm F, Bruley DF, Harrison DK, eds. Oxygen Transport to Tissue XXXIV. New York: Springer; 2013: 211-216

42 Simchon S, Jan K-M, Chien S. Influence of reduced red cell deformability on regional blood flow. Am J Physiol 1987;253(4, Pt 2): H898-H903
43 Chien S. Rheology in the microcirculation in normal and low flow states. Adv Shock Res 1982;8:71-80

44 Maeda N. Erythrocyte rheology in microcirculation. Jpn J Physiol 1996;46(01):1-14

45 Maino A, Rosendaal FR, Algra A, Peyvandi F, Siegerink B. Hypercoagulability is a stronger risk factor for ischaemic stroke than for myocardial infarction: a systematic review. PLoS One 2015;10 (08): $\mathrm{e} 0133523$

46 Kolominsky-Rabas PL, Weber M, Gefeller O, Neundoerfer B, Heuschmann PU. Epidemiology of ischemic stroke subtypes according to TOAST criteria: incidence, recurrence, and long-term survival in ischemic stroke subtypes: a population-based study. Stroke 2001; 32(12):2735-2740

47 Hutcheon JA, Chiolero A, Hanley JA. Random measurement error and regression dilution bias. BMJ 2010;340:c2289

48 Pepys MB, Hirschfield GM. C-reactive protein: a critical update. J Clin Invest 2003;111(12):1805-1812 